\title{
Ribosomal DNA internal transcribed spacer 2 sequence analysis and phylogenetic comparison of seven cockroach species in northwestern Iran
}

\author{
Mostafa Farmani ${ }^{1,2}$, Hamidreza Basseri ${ }^{3}$, Behzad Norouzi ${ }^{4}$ and Saber Gholizadeh ${ }^{1,2^{*}}$ (1)
}

\begin{abstract}
Objectives: The current study was conducted to identify cockroach species (Blattodea) of northwestern Iran in public places using morphological characteristics and ribosomal DNA internal transcribed spacer 2 (rDNA-ITS2). Sequences were analyzed with Basic Local Alignment Search Tool (BLAST) searches, Neighbor-Joining methods based on and Tamura-Nei phylogenetic analyses. In addition, eight cockroach rDNA-ITS2 sequences from China, India, Iran and the United States obtained from GenBank were compared to those obtained in this study.

Results: Specimens collected in Iran were identified as Periplaneta americana (L.), Shelfordella lateralis (Walker), Blatta orientalis (L.) (Blattodea: Blattidae), Blattella germanica (L.), Supella longipalpa (F.) (Blattodea: Ectobiidae), Polyphaga aegyptiaca (L.), and Polyphaga saussurei (Dohrn) (Blattodea: Corydiidae). rDNA-ITS2 nucleotide sequence analysis showed $100 \%$ similarity between P. aegyptiaca and P. saussurei species collected from Iran despite morphological differences. However, ITS2 sequence of P. americana submitted from China showed 30.49-31.71\% difference to $P$. americana sequences from Iran and the United States. The results highlight the importance of morphological identification of cockroach species before conducting molecular techniques.
\end{abstract}

Keywords: rDNA-ITS2, Phylogeny, Blattidae, Ectobiidae, Corydiidae

\section{Introduction}

Cockroach is one of the most important urban pests in the world. They are mostly known for their role in allergies and can transmit some diseases to human [1-4].

Mirzayans reported 24 species of cockroaches in Blattidae, Ectobiidae, and Corydiidae families in Iran [5]. More recent surveys by Hanafi-Bojd, Sadaghiyani and Hashemi-Aghdam, Oshaghi reported 3 families, 14 genera, and 26 species in Iran [6, 7]. Two more species are Parcoblatta sp. [8], and Polyphaga sp. [7].

Mitochondrial and nuclear molecular markers are used for the precise identification of cockroach species and

\footnotetext{
*Correspondence: sabergholizadeh@yahoo.com

${ }^{1}$ Cellular and Molecular Research Center, Urmia University of Medical Sciences, Urmia, Iran

Full list of author information is available at the end of the article
}

their phylogenetic relationship [9]. rDNA-ITS2 might be appropriate for mushrooms and Diptera, but it does not mean that it is appropriate for (some) cockroaches [10-12]. Ribosomal DNA has not been used frequently for identification of cockroach species [13]. ITS2 length varies among different cockroach species [13], and ITS2 sequences are often more polymorphic between species than within species [14]; therefore, it could be useful for molecular identification of sibling species [15].

A better understanding of the cockroach ecology and taxonomy is essential for the successful pest control program. Thus, a routine survey of the cockroach population in northwestern Iran will greatly contribute to the success of the control program. This study was designed to identify morphologically and molecularly cockroach species in northwestern Iran and to determine the phylogenetic relationships (using rDNA-ITS2 sequences) 
among these species. New set of primers were designed as universal primers to amplification of rDNA-ITS2 fragment in cockroach species.

\section{Main text \\ Methods \\ Cockroach collection and morphological identification}

The majority of cockroaches used in this study were collected manually by searching their shelters using flashlight at night from 30 locations in Urmia, Iran $\left(37^{\circ} 33^{\prime} 19^{\prime \prime} \mathrm{N} 45^{\circ} 04^{\prime} 21^{\prime \prime} \mathrm{E}\right)$ in $2013-2015$. Plastic bottle traps and sticky cards $(\mathrm{n}=10$ in each location) baited with sugar, biscuits and dried breads were used to collect cockroaches in houses, hospitals, dormitories and landfill. Trappings were conducted from 6:00 p.m. to 6:00 a.m. in 2015. Cockroaches were captured from the plastic bottle traps and transferred to containers individually. The inner surface of the plastic bottle traps was coated with butter to prevent escape.

Adult cockroaches were killed at $-20{ }^{\circ} \mathrm{C}$ and identified using morphological keys $[5,6]$. Voucher specimens were deposited in the Entomology Laboratory at the School of Public Health (SPH), Urmia Medical Sciences University (UMSU), Urmia, Iran.

\section{DNA extraction}

Genomic DNA was extracted from the thorax of individual cockroach species, stored in $70 \%$ ethanol, using YTA Genomic DNA Extraction Mini Kit (Yekta Tajhiz Azma, Tehran, Iran). Based on manufacturer's instruction, a 25-mg tissue sample was removed from the thorax of each cockroach with a surgical blade and homogenized in $200 \mu \mathrm{L}$ TG1 buffer by grinding with a micropestle containing liquid nitrogen. After adding $20 \mu \mathrm{L}$ proteinase $\mathrm{K}$, the mixture was incubated at $60^{\circ} \mathrm{C}$ for $1-2 \mathrm{~h} .200 \mu \mathrm{L}$ of TG2 buffer was added and re-incubated for $10 \mathrm{~min}$ at $70{ }^{\circ} \mathrm{C}$, after which $200 \mu \mathrm{L}$ cold ethanol was added. The mixture was transferred to TG Mini Column and centrifuged for $1 \mathrm{~min}$ at $8000 \mathrm{rpm}$. The flow-through was discarded and TG Mini Column was transferred to a new Collection Tube. DNA was washed with $500 \mu \mathrm{L}$ of W1 and $750 \mu \mathrm{L}$ of wash buffers by centrifuging for $1 \mathrm{~min}$ at $14,000 \mathrm{rpm}$. Total DNA was eluted to the elution tube by adding $100 \mu \mathrm{L}$ elution buffer or $\mathrm{ddH}_{2} \mathrm{O}(\mathrm{pH}$ 7.5-9.0) and stored at $4{ }^{\circ} \mathrm{C}$ or $-20^{\circ} \mathrm{C}$ until use [16].

\section{Primer designing and $P C R$ amplification}

Cockroach-specific primers (5.8S TGGGTCGATGAA GAACGC and 28S ATTCAGCGGGTAGTCTCG) were designed based on cockroach rDNA sequences available in the GenBank (GenBank ID: AF005243, KF899831, and EU306665) using the softwares Gene Runner (Hastings
Software Inc. 1994) and Standard Nucleotide BLAST [17].

PCR reactions of ITS2 fragment were performed in a total volume of $25 \mu \mathrm{L}$ master mix. Each reaction contained $2 \mu \mathrm{L}$ genomic DNA, $12.5 \mu \mathrm{L}$ PCR Master Mix (Yekta Tajhiz Azma, Tehran, Iran), $0.2 \mu \mathrm{L}$ Taq polymerase, $1 \mu \mathrm{L}$ each primer (forward and reverse), and $8.3 \mu \mathrm{L}$ $\mathrm{ddH}_{2} \mathrm{O}$. The PCR amplification profile was set as follows: initial template denaturation at $95^{\circ} \mathrm{C}$ for $5 \mathrm{~min}$, followed by 30 cycles of denaturation at $95^{\circ} \mathrm{C}$ for $1 \mathrm{~min}$, annealing at $54{ }^{\circ} \mathrm{C}$ for $1 \mathrm{~min}$, and extension at $72{ }^{\circ} \mathrm{C}$ for $1 \mathrm{~min}$, with a final 10-min elongation step at $72{ }^{\circ} \mathrm{C}$. The PCR products $(5 \mu \mathrm{L})$ were run on $1.5 \%$ agarose gel stained with safe stain (Yekta Tajhiz Azma Co. Cat No. YT0001, Tehran, Iran), and bands were visualized by UV trans-illumination (Syngene GBOX/EF, Cambridge, England). A total of 30 specimens were subjected to sequencing with an ABI377 automatic sequencer (SeqGen, Torrance, Canada), using the same amplification primer.

Eight rDNA-ITS2 sequences of Blatta orientalis L. (GenBank IDs: EU306665, KF899833), Periplaneta americana (L.) (KF899831, AF321248, and AJ577262) and Blattella germanica L. (KF899832, AF005243, and AF321244) from China [18], India [19], central Iran [20] and the United States [13], belong to seven species collected in the current study, were also obtained from GenBank. These sequences and 30 sequences of the seven Iranian cockroach species were aligned, and a Mantis religiosa L. (Mantodea: Mantidae) ITS2 sequence (GenBank ID: AY859585) [21] was used as out-group. The sequences obtained from GenBank were compared to the sequences obtained in this study.

\section{Sequence analysis}

The acquired ITS2 sequences were annotated according to the previously submitted sequences using the ITS2 annotation tool, version 3.0.13 [22]. For rDNAITS2 sequence alignment and phylogenetic analyses two online software programs, BLAST [23], Clustal Omega [24], and an offline software, Molecular Evolutionary Genetics Analysis 7 (MEGA7) [25], were utilized.

The phylogenetic tree was constructed using both maximum likelihood and neighbor-joining methods based on Tamura-Nei model $[26,27]$. The percentages of replicating trees in which the associated taxa clustered together in the bootstrap test (1000 replicates) are indicated for each branches [28].

\section{Results}

Three-hundred-twenty-one cockroaches representing 6 genera and 7 species were collected in the current study (Table 1). Blattella germanica was the most frequently collected species, whereas Polyphaga aegyptiaca L. and 
Table 1 Species and frequency of cockroaches collected from Urmia, West Azerbaijan Province, Iran

\begin{tabular}{llc}
\hline Family & Species & $\begin{array}{l}\text { Number collected } \\
\text { (\% total collected) }\end{array}$ \\
\hline Blattidae & Blatta orientalis L. & $23(7.2)$ \\
& Shelfordella lateralis Walker & $62(19.3)$ \\
& Periplaneta americana L. & $57(17.8)$ \\
Ectobiidae & Supella longipalpa F. & $19(5.9)$ \\
& Blattelagermanica L. & $146(45.5)$ \\
Corydiidae & Polyphaga aegyptiaca L. & $7(2.2)$ \\
& Polyphaga sausserei Dohrn & $7(2.2)$ \\
\hline
\end{tabular}

Polyphaga saussurei Dohrn were the least frequently collected.

The amplified fragment size using designed primers was 340 bp in Supella longipalpa (F.), 385 bp in $P$. aegyptiaca and $P$. saussurei, $412 \mathrm{bp}$ in $P$. americana and S. lateralis, 418 bp in B. orientalis, and 592 bp in B. germanica. Results from ITS2 annotation tool showed different ITS2 sizes, varying from 179 bp in S. longipalpa to $431 \mathrm{bp}$ in B. germanica. Also, the 128-bp upstream and 33-bp downstream of ITS2 sequences were $5.8 \mathrm{~s}$ and $28 \mathrm{~s}$, respectively. These sequences were submitted to GenBank under the accession numbers KY817789 to KY817818, representing the first records for some species in Iran and the world.

The numbers of cockroach rDNA-ITS2 sequences deposited in GenBank were low, which limited our ability to conducted more BLAST analyses and comparisons to species collected in Iran. Sequence similarity within species of $B$. germanica, S. lateralis, $B$. orientalis, S. longipalpa, P. aegyptiaca, and P. saussurei, collected from Urmia, was $99.55-100 \%$, whereas the identity within $P$. americana sequences was 97.61$100 \%$. BLAST comparison of B. germanica sequences from Iran and the United States showed 99.53-100\% similarity. The sequence of $B$. orientalis from India was only $45.28 \%$ similar to those isolated from B. orientalis collected in this study, but showed more similarity (98.36-98.83\%) to those of B. germanica from GenBank (KF899832, AF005243, and AF321244). The similarity between sequences of $P$. americana from Iran (KF899831) and the United States (AF321248) was $97.21-100 \%$, but the similarities of the Iranian and American sequences to the Chinese sequence (AJ577262) were $68.70 \%$ and $68.29-69.51 \%$, respectively. Moreover, the sequence sizes of rDNA-ITS2 region in $P$. americana (from Iran, the United States, and China) and P. saussurei (from Iran) were the same as S. lateralis (from Iran) and P. aegyptiaca (from Iran), i.e. $251 \mathrm{bp}$ and $224 \mathrm{bp}$, respectively. Sequence similarity analysis showed $88.80 \%$ identity between $P$. americana and S. lateralis, whereas it was $99.55 \%$ in P. saussurei and $P$. aegyptiaca.

Phylogenetic analysis revealed that the seven Iranian cockroach species were clustered into two main branches (Fig. 1). Periplaneta americana, B. orientalis and S. lateralis, members of Blattidae, were clustered in the first branch. However, B. germanica and S. longipalpa were placed in two separate plural. Polyphaga aegyptiaca and $P$. saussurei were clustered in the same clade and branch (Fig. 1). Interestingly, B. orientalis from India (EU306665) was placed in the B. germanica cluster, whereas $P$. americana from China (AJ577262) was clustered in a separate clade near Blattidae.

\section{Discussion}

The cockroach fauna in Urmia is unknown, it is not included in Iranian checklist of cockroaches [5, 7].

Several sequences of species obtained in this study were not completely similar to those of the same species obtained from GenBank. The sequence of $B$. orientalis from India (EU306665) was similar to that of B. germanica obtained in this study. While, these two species belongs to two different cockroach families with high distinct in morphology, rDNA-ITS2 size and sequence. Phylogenetic analysis also revealed that sequence of P. americana from China (AJ577262) was clustered in a separate clade from $P$. americana from Iran and the United States. It seems those are morpho-taxonomic mistakes in cockroach species identification. These mistakes suggested it is good advice to ensure cockroach species identification before any molecular analysis and depositing the sequences to the GenBank.

This study showed that rDNA-ITS2 sequence analysis of $P$. aegyptiaca and P. saussurei is $100 \%$ similar in size (224 bp) and sequence. A high degree of rDNA-ITS2 sequence and size similarity, despite morphological differences in body shape and color, also had been reported between Anopheles hyrcanus (Pallas) and Anopheles pseudopictus (Grassi) (Diptera: Culicidae) (Ponçon et al. 2008). Such similarity suggested that $P$ aegyptiaca and $P$. saussurei may be the same species although they are considered separate species based on morphological characteristics. However, only ITS2 marker is not sufficient for these conclusion and need to study with other molecular markers.

In the phylogenetic tree, three Iranian cockroach families were classified into four clades. It was notable that B. germanica and S. longipalpa belongs to Ectobiidae clustered in two different clades. Recent phylogenetic analyses of cockroaches showed that Ectobiidae are paraphyletic [29-31]. 


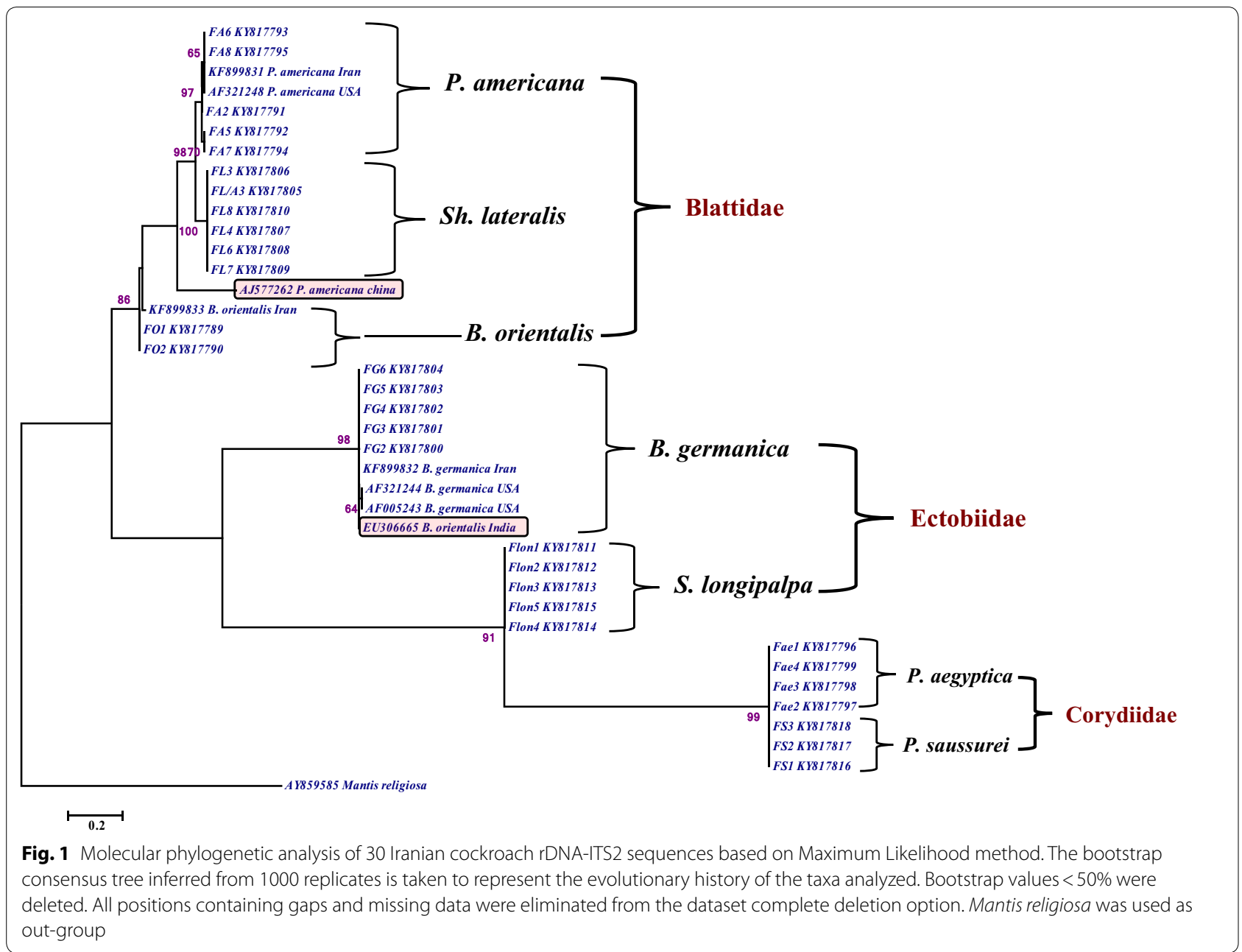

\section{Conclusions}

We would like to emphasize the importance of considering both morphological and molecular data in identifying field collected specimens of cockroaches before designing, implementing and evaluating control programs. Incorrect identification of insect species may sometimes lead to the application of incorrect vector control strategies [32] and can have significant consequence to the efficiency of vector and urban pest management programs.

\section{Limitations}

The major concerns within this study is the use of only one genetic marker (ITS2) instead of multiple markers such as cytochrome oxidase I (COI) and cytochrome oxidase II (COII) (because of founding limitations for MSc student project). The current study were conducted in Urmia district among seven cockroach species, however, the efficiency of new primers need to evaluate on other cockroach species in large areas.

\section{Abbreviations}

rDNA-ITS2: ribosomal DNA internal transcribed spacer 2; BLAST: Basic Local Alignment Search Tool; MEGA7: Molecular Evolutionary Genetics Analysis version 6.0; EMBL: European Molecular Biology Laboratory; DDBJ: DNA Data Bank of Japan; COI: cytochrome oxidase l; COIl: cytochrome oxidase II.

\section{Authors' contributions}

Conceived and designed the experiments: SG and MF. Performed the experiments: MF and HB. Analyzed the data: SG. Contributed reagents/materials/ analysis tools: SG, MF and BN. Wrote the paper: SG, MF, and HB. All authors read and approved the final manuscript.

\section{Author details}

${ }^{1}$ Cellular and Molecular Research Center, Urmia University of Medical Sciences, Urmia, Iran. ${ }^{2}$ Medical Entomology Department, School of Public Health, Urmia University of Medical Sciences, Urmia, Iran. ${ }^{3}$ Medical Entomology Department, School of Public Health, Tehran University of Medical Sciences, Tehran, Iran. ${ }^{4}$ Research Center of Health and Environment, Guilan University of Medical Sciences, Rasht, Iran.

\section{Acknowledgements}

Funding of this project (Number 1394-01-34-1938) was provided by Cellular and Molecular Research Center, Urmia University of Medical Sciences, Urmia, Iran. 


\section{Competing interests}

The authors declare that they have no competing interests.

\section{Availability of data and materials}

Data supporting of this article are included within the article and additionalfile.

\section{Consent for publication}

Not applicable.

\section{Ethics approval and consent to participate}

Not applicable.

\section{Funding}

Funding of this project (Number 1394-01-34-1938) was provided by Cellular and Molecular Research Center, Urmia University of Medical Sciences, Urmia, Iran

\section{Publisher's Note}

Springer Nature remains neutral with regard to jurisdictional claims in published maps and institutional affiliations.

Received: 18 December 2018 Accepted: 14 January 2019

Published online: 23 January 2019

\section{References}

1. Moiser B. Hansen's disease (leprosy) and cockroaches. East Afr Med J. 1947;24(6):230-6.

2. Burgess NR, Chetwyn KN. Association of cockroaches with an outbreak of dysentery. Trans R Soc Trop Med Hyg. 1981;75(2):332-3.

3. Fotedar R, Shriniwas UB, Verma A. Cockroaches (Blattella germanica) as carriers of microorganisms of medical importance in hospitals. Epidemiol Infect. 1991;107(1):181-7.

4. Londres MI, Sarinho FW, Miranda PJ, Solé D, Sarinho E. Allergy to cockroaches: challenges in diagnosis. J Clin Lab. 2011;57(11-12):969-74.

5. Mirzayans H. Fauna of Iranian cockroaches:(Orthopteroidea: Blatt Aria). J Entomol Soc Iran. 1986;9:1-134.

6. Hanafi-Bojd AA, Sadaghiani S. Cockroaches of Iran. Tehran: Tehran University of Medical Sciences Press; 2009.

7. Hashemi-Aghdam SS, Oshaghi MA. A checklist of Iranian cockroaches (Blattodea) with description of Polyphaga sp as a new species in Iran. J Arthropod Borne Dis. 2015;9(2):161-75.

8. Fathpour H, Emtiazi G, Ghasemi E. Cockroaches as reservoirs and vectors of drug resistant Salmonella spp. Iran Biomed J. 2003;7(1):35-8.

9. Maekawa K, Matsumoto T. Molecular phylogeny of cockroaches (Blattaria) based on mitochondrial COll gene sequences. Syst Entomol. 2000;25(4):511-9.

10. Smith MA, Fisher BL, Hebert PD. DNA barcoding for effective biodiversity assessment of a hyperdiverse arthropod group: the ants of Madagascar. Philos Trans R Soc B. 2005;360(1462):1825-34

11. Dentinger BT, Didukh MY, Moncalvo J-M. Comparing COI and ITS as DNA barcode markers for mushrooms and allies (Agaricomycotina). PLoS ONE. 2011;6(9):e25081.

12. Gholizadeh S, Djadid ND, Nouroozi B, Bekmohammadi M. Molecular phylogenetic analysis of Anopheles and Cellia subgenus anophelines (Diptera: Culicidae) in temperate and tropical regions of Iran. Acta Trop. 2013;126(1):63-74.

13. Mukha D, Wiegmann BM, Schal C. Evolution and phylogenetic information content of the ribosomal DNA repeat unit in the Blattodea (Insecta). Insect Biochem Mol Biol. 2002:32(9):951-60.
14. Manonmani A, Townson H, Adeniran T, Jambulingam P, Sahu S, Vijayakumar T. rDNA-ITS2 polymerase chain reaction assay for the sibling species of Anopheles fluviatilis. Acta Trop. 2001:78(1):3-9.

15. Musters W, Boon K, van der Sande CA, van Heerikhuizen H, Planta RJ. Functional analysis of transcribed spacers of yeast ribosomal DNA. EMBO J. 1990;9(12):3989-96.

16. Firooziyan S, Dinparast Djadid N, Gholizadeh S. Speculation on the possibility for introducing Anopheles stephensi as a species complex: preliminary evidence based on odorant binding protein 1 intron I sequence. Malar J. 2018;17(1):366.

17. Altschul SF, Gish W, Miller W, Myers EW, Lipman DJ. Basic local alignment search tool. J Mol Biol. 1990;215(3):403-10.

18. Ji YJ, Zhang DX, He LJ. Evolutionary conservation and versatility of a new set of primers for amplifying the ribosomal internal transcribed spacer regions in insects and other invertebrates. J Mol Ecol Notes. 2003:3(4):581-5.

19. Kuracha MR, Tamalampudi VR, Duvvuri B, Duvvuri SK, Nagaraja Rao P: GenBank: Accession no. EU306665. https://www.ncbi.nlm.nih.gov/genba $n k / .2007$.

20. Rafie G, Oshaghi MA, Shiravi A, Shayeghi M, Hashemi-Aghdam SS, Bakhshi H: GenBank: Accessions no. KF899831-KF899833. https://www.ncbi.nlm. nih.gov/genbank/. 2013.

21. Mallatt J, Giribet G. Further use of nearly complete $28 \mathrm{~S}$ and $18 \mathrm{~S}$ rRNA genes to classify Ecdysozoa: 37 more arthropods and a kinorhynch. J Mol Phylogenet Evol. 2006;40(3):772-94.

22. Koetschan C, Forster F, Keller A, Schleicher T, Ruderisch B, Schwarz R, Muller T, Wolf M, Schultz J. The ITS2 Database III-sequences and structures for phylogeny. Nucleic Acids Res. 2010;38(Database issue):D275-9.

23. Chen Y, Ye W, Zhang Y, Xu Y. High speed BLASTN: an accelerated MegaBLAST search tool. J Nucleic acids Res. 2015;43(16):7762-8.

24. Sievers F, Higgins DG. Clustal Omega, accurate alignment of very large numbers of sequences. In: Multiple sequence alignment methods. New York: Springer; 2014. p. 105-16.

25. Tamura K, Dudley J, Nei M, Kumar S. MEGA4: molecular evolutionary genetics analysis (MEGA) software version 40. Mol Biol Evol. 2007:24(8):1596-9.

26. Saitou N, Nei M. The neighbor-joining method: a new method for reconstructing phylogenetic trees. Mol Biol Evol. 1987:4(4):406-25.

27. Tamura K, Nei M. Estimation of the number of nucleotide substitutions in the control region of mitochondrial DNA in humans and chimpanzees. Mol Biol Evol. 1993;10(3):512-26.

28. Felsenstein D, Carney WP, lacoviello VR, Hirsch MS. Phenotypic properties of atypical lymphocytes in cytomegalovirus-induced mononucleosis. J Infect Dis. 1985;152(1):198-203.

29. Djernæs M, Klass KD, Eggleton P. Identifying possible sister groups of Cryptocercidae + Isoptera: a combined molecular and morphological phylogeny of Dictyoptera. Mol Phylogenet Evol. 2015;84:284-303.

30. Legendre F, Nel A, Svenson GJ, Robillard T, Pellens R, Grandcolas P. Phylogeny of Dictyoptera: dating the origin of cockroaches, praying mantises and termites with molecular data and controlled fossil evidence. PLoS ONE. 2015:10(7):e0130127.

31. Wang Z, Shi Y, Qiu Z, Che Y, Lo N. Reconstructing the phylogeny of Blattodea: robust support for interfamilial relationships and major clades. Sci Rep. 2017;7(1):3903.

32. Van Bortel W, Harbach RE, Trung HD, Roelants P, Backeljau T, Coosemans M. Confirmation of Anopheles varuna in Vietnam, previously misidentified and mistargeted as the malaria vector Anopheles minimus. Am J Trop Med Hyg. 2001;65(6):729-32. 